\author{
Military Technical College \\ Kobry El-Kobbah, \\ Cairo, Egypt.
}

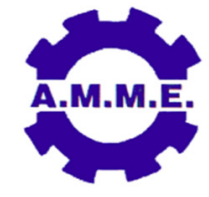

\title{
USE OF GT-SUITE TO STUDY PERFORMANCE DIFFERENCES BETWEEN INTERNAL COMBUSTION ENGINE (ICE) AND HYBRID ELECTRIC VEHICLE (HEV) POWERTRAINS
}

\author{
M. S. Asfoor ${ }^{*}$, A. M. Sharaf ${ }^{\star *}$ and S. Beyerlein ${ }^{* *}$
}

\begin{abstract}
Hybrid Electric Vehicles (HEVs) are receiving a great deal of interest around the world due to not only their promise of higher energy efficiency and reduced highway emissions, but also their ability to overcome the range limitations inherent in a purely electric automobile. In a hybrid powertrain, energy is stored as a petroleum fuel and in an electrical storage device, such as a battery pack, and is converted to mechanical energy by an internal combustion engine (ICE) and an electric motor (EM), respectively. The EM is used to improve energy efficiency and vehicle emissions while the ICE provides extended range capability.
\end{abstract}

Computer simulation is a valuable tool for analyzing hardware components and predicting vehicle performance with different powertrain configurations. In this work a traditional ICE operated vehicle is compared to several hybrid versions of the same vehicle, all modeled using GT-Suite. A variety of standard driving cycles are considered, among them the Federal Test Procedure (FTP) for city driving, the Highway Fuel Economy Test (HWY), the high acceleration aggressive driving schedule (US06) that is often identified by the Supplemental FTP, and the New European Driving Cycle (NEDC). This study considers a rule-based energy management strategy for power splitting in the hybrid powertrain models. ICE only and hybrid modes are compared based on average as well as instantaneous performance. The overall fuel economy, energy consumption and losses in the ICE and HEV powertrain models are monitored and compared based on average performance, and a comprehensive energy analysis is performed to track energy sources and sinks. The paper results reveal the benefits of HEVs in terms of reduced fuel energy consumption and improved fuel economy.

\section{KEY WORDS}

Hybrid Electric Vehicles, Fuel Economy, Vehicle Modeling, Driving Cycles, GT-Suite

\footnotetext{
* Department of Mechanical Engineering, University of Idaho, Idaho, USA.

** Associate Professor, Egyptian Armed Forces.

*** Professor, Department of Mechanical Engineering, University of Idaho, Idaho, USA.
} 


\section{INTRODUCTION}

Transportation-related energy consumption and air quality degradation have been a major concern for years. Numerous technologies and strategies have been developed and deployed to address these energy and environment issues. Among those, advanced vehicle technologies, HEVs and Electric Vehicles (EVs), are very promising in improving fuel efficiency of motor vehicles.

HEVs have existed since before 1900, but interest in these vehicles has grown substantially only in the last 20 years. The main reason for this interest is the expectation that HEVs represent a short-term approach for improving fuel efficiency and reducing pollutant emissions. It is commonly known that, a Hybrid vehicle comprises two or more power sources in the drivetrain. There are many different layouts of HEVs, but only the gasoline-electric hybrids are currently commercially available. HEVs could be classified according the arrangement of input power as: Series, Parallel and Combined HEV. Each of these configurations has different operating modes where power flow is controlled through a vehicle supervisory controller. These operating modes differ from electric only, engine only, electric power assist, battery charging and regenerative braking modes [1-2] .

Generally, two main approaches are usually used in vehicle simulation. These approaches are usually categorized according to the propagation of power flow inside the simulators. So, Forward-facing modeling simulates the physical behavior of each component with control instruction, handles state changes, and generates vehicle performance as output. While Backward-facing modeling takes the assumption that the vehicle meets the target performance, and calculates the component states. Backward-facing approach is beneficial in simplicity and computation cost, though they are usually used to define trends, while forward-facing approach is advantageous in utilizing performance details, though allow selection of powertrain configurations as well as development of controls that will later be implemented in the vehicles [3-4].

Several computer programs have been developed to describe the operation of hybrid electric powertrains. Among these simulation tools, MATLAB/Simulink [5-6], ADVISOR [7], PSAT [8] and Autonomie from the US Department of Energy, GTSuite by Gamma Tech., and others have been widely used in the field of vehicle research. All of these software packages are introduced to simulate issues related to EVs and HEVs design such as energy efficiency, fuel economy, vehicle emissions, power plant configurations, component sizing, Energy Management System (EMS), and optimization of important parameters [9].

GT- Suite is a product of Gamma Technologies (GTI), a specialist software company which is solely focused on the engine and vehicle industry, practically all leading engine makers and suppliers have chosen GT- Suite because it is supplied as an allinclusive package with many valuable productivity tools that are included. These tools increase user efficiency, where it offers a versatile set of tools for simulation of vehicles with conventional, HEV or EV drivelines, as well as the control systems and strategies that are keys to the operation of these vehicles. It handles, in a single tool, a wide variety of vehicle and engine technical applications. It is a unique tool, which provides the ability to execute integrated simulations of the entire vehicle and engine system. Such simulations are an industry trend that is gaining in importance and they 
constitute the next frontier in Computer Aided Engineering (CAE) applications. GTSuite has long been recognized for its high degree of accuracy in predicting the behavior of complex engine related phenomena.

This work demonstrates a comparative analysis between a traditional ICE operated vehicle and a three different hybrid powertrain developed versions of the same vehicle. The GT-Suite software is used as an environment where all models undergo different driving cycles which have great impact on the vehicle's performance in terms of overall energy consumption, and fuel economy. The models predict vehicle performance criteria including fuel economy, maximum \& average acceleration, maximum \& average speed, vehicle and driveline energy losses. The results of the model show the significant benefits of HEVs in terms of reducing fuel consumption, lowering running costs and improving driving performance and ease of use.

\section{MODELS DESCRIPTION}

In this study, the GT-Suite software is used for comparison between an ICE only vehicle and different hybrid architectures with the same engine. Separate models are investigated with rule-based EMS performing the same driving cycles. This includes a conventional vehicle with ICE only, Series, Parallel and Series-Parallel hybrid configurations. Typical values of road resistances are used while running the models. The presented simulation model is dynamic, modular, forward-type simulation and consists of a driver sub-model trying to follow a predetermined speed profile.

\section{Conventional ICE Vehicle Model}

The conventional drivetrain is powered with a $2.0 \mathrm{~L}$ gasoline engine which is modeled as a map-based engine performance representing power output and friction, fuel consumption, and other characteristics. The engine maps for these quantities are specified as a function of engine speed and load. In addition, the ICE controller is used to simulate engine control functions such as idling and fuel cut off, this object is recommended for applications where maximizing fuel economy is important.

The engine torque which is applied at the crankshaft is modeled by a look-up map and varies with accelerator pedal positions and engine speeds. The driver module is incorporated to represent the driver actions that control the accelerator pedal, brake pedal, and transmission gear number during driveway and shifting. The 'Driver Controller' is a model based controller that is typically used when performing dynamic driving cycle analysis. The model consists of a feed forward component which calculates the engine load torque required to correlate the desired vehicle speed or acceleration. For this calculation, the driver controller extracts key information from the vehicle drivetrain. Once the reference load torque is calculated, a standard PI controller is used to correct the demanded load from the engine or brakes to minimize the remaining error between target and instantaneous values.

A Transmission Controller is used to represent automatic transmission control logic for gear selection. This component is used to recall specified transmission shift logic to determine the desired transmission gear number. A Lockup clutch connection is 
used to model the action of a friction clutch between the engine and transmission. An Environment module is used to specify the ambient air conditions that affect the aerodynamic force on the vehicle. Several attributes are used to determine the air density including relative humidity, ambient air temperature and pressure. The wind velocity and direction are used to determine the effective vehicle-air velocity. The density and effective air velocity are used in drag and lift force calculations. A Road module is used to model the road properties that affect vehicle dynamics including road grad, elevation, curvature radius, and rolling resistance. The conventional power plant model is shown in Fig. 1, and Table 1 shows the ICE and vehicle specifications used in the model.

\section{Hybrid Electric Vehicle Models}

Traditionally, HEVs were classified into two basic layouts, series and parallel. With the introduction of some HEVs offering the features of both the series and parallel hybrids, the classification has been extended to three kinds, series, parallel and series-parallel. Fig. 2 shows the corresponding functional block diagrams, in which the electrical and mechanical links are bidirectional while the hydraulic link is unidirectional $[10,11,12]$.

In addition to the same 2.0L gasoline ICE, typical components which are commonly found in HEV configurations are developed and presented. These include mapbased modules for traction motor and generator. The model considers the specification of electrical power request or mechanical brake power or torque, plus an electrical-to-mechanical (or vice versa) power conversion efficiency and mechanical friction characteristic. The traction motor and generator are controlled via two electro-mechanical controllers which are programmed to follow the control strategy rules. The brake power necessary to follow a driving cycle is calculated by a power demand template, which is a model based controller. It calculates the necessary tractive power or axle torque required for a targeted vehicle speed or acceleration including tire rolling resistance, aerodynamic drag, road curvature and road grade effects.

A model for the battery pack of a capacity of $23 \mathrm{Ah}$ is developed and represented by charge and discharge lookup maps for internal resistance and open-circuit voltage. The battery model calculates the State of Charge (SOC) which is defined as the level of electric capacity remaining in the battery. The SOC is calculated based on the power being drawn from or supplied to the electric circuit, depending on the direction of the current. An inverter is used in conjunction with the battery pack template to ensure the maximum discharge and charge power limits of the battery are not exceeded when it is connected to the electrical or electromagnetic component(s). The inverter is a controls-based compound that outputs the electrical power limits based upon the maximum available discharge and charge power as given in Table 2.

A Braking Module is introduced to calculate the brake pedal position based upon the desired braking power, and maximum torque capability of the brakes. This module is equipped with a braking control strategy that allow for energy regeneration according to the vehicle operating mode and under the supervision of the main control strategy. 
The supervisory controller acts as a high-level vehicle control system that coordinates the overall powertrain to satisfy certain performance target, and serves as an energy management unit. The EMS refers to a control algorithm that determines the proper power/torque level to be generated, and its split between the EM and the ICE while satisfying the power demand from the driver and maintaining sufficient energy in the energy storage device. The primary objective of the EMS is to coordinate the power flow between the energy carriers and the environment in response to the driver's power demand, while improving fuel economy, reducing exhaust emissions and maintaining various subsystems in their desired states without affecting vehicle performance constraints, such as acceleration and gradeability, ensuring seamless operation of the drivetrain.

\section{Series HEV model}

A Series HEV configuration dynamically runs different driving cycles is modeled, where the power demand request is actuated on the traction motor, which is also used for regenerative braking. The battery discharge limiter ensures that the connected battery with the traction motor and generator is not overdrawn. The generator acts as an integrated starter/generator so the ICE can be shutoff and turned back on. The control strategy is a "thermostat on-off" strategy. The ICE is shutoff when the battery SOC is between certain limits during battery discharge. When the battery SOC decreases below 0.5, the starter/generator turns the engine on and the generator charges the battery. The ICE is run at its lowest Brake Specific Fuel Consumption (BSFC) speed for maximum efficiency. When the battery SOC is above 0.7 , the engine is shut back off. The Series HEV model is shown in Fig. 3.

\section{Parallel HEV model}

A torque coupling-type parallel HEV is modeled as a basic model which could be further modified into two configurations according to the location of the traction motor. A pre-transmission parallel hybrid, in which the traction motor is connected to the ICE prior to the transmission, and a post-transmission parallel hybrid, in which the traction motor is connected to either the driven axle, after the final drive, or the non-driven axle. In the presented parallel hybrid configuration, there is only one traction motor that acts as a propulsion motor or generator for battery charging as shown in Fig. 4.

The vehicle has the features of motor drive away, idle stop and regenerative braking. The control system is setup in such a way that, anytime the vehicle stops for over a second, the engine is switched off. When the vehicle begins to move again, the motor powers the driveline and also cranks up the engine. Once the engine reaches a preset speed, the motor actuator position is set to zero. Until the engine reaches the preset speed, it is merely motored and not fueled hence increasing fuel economy. The regenerative braking works by setting a negative actuator position when the brake pedal position is positive. Since the regenerative brakes may not be able to provide enough braking torque for every braking event, friction brakes are also provided. There is a charging circuit that sets a negative actuator position for the EM depending on the battery SOC. 


\section{Series-Parallel HEV model}

The model of Series-Parallel HEV configuration consists of a combination of ICE and generator subsystem using a planetary gear set to connect each other and an electric drive subsystem, constructing two driving power sources, each has its own controller. In order to ensure the proper functionality of all controllers together to meet the required driver's power demand and provide an efficient onboard energy usage, a supervisory controller is required to maintain the vehicle at its most efficient operating conditions by managing the power among the two driving power sources.

This architecture combines the advantages of both series and parallel powertrains. The model utilizes the same map-based 2.0L gasoline ICE with some modifications made to its controller to allow the execution of the main control strategy rules. The necessary tractive power to follow a certain driving cycle is calculated by the power demand template. The torque request includes the necessary torque to accelerate the vehicle and its cargo mass as well as the vehicle system inertias.

The traction motor and the generator are configured such that by closing a single clutch the architecture can be changed from series to parallel. Clutch_1 is used to model the action of a dry clutch between two 1-D rotational mechanical assemblies, naming traction motor shaft and driveline input shaft. Clutch_2 is used to model a simple clutch which is either engaged or disengaged by applying a constraint to set the two angular velocities on either side of the clutch equal to each other. Fig. 5 shows the configuration of the developed series-parallel HEV power plant model.

There are six independent input parameters that affect the control strategy as follow:

- The necessary tractive power demand to follow a given driving cycle.

- Battery status which is determined via the battery SOC and helps to switch between charging and discharging modes according to the prescribed limits.

- Vehicle and engine speeds which are used to select of the best operating mode.

- Engine max power at current speed which helps in combination with the power demand in determination of the amount of power required from the EM.

- Traction motor speed aids in determination of the maximum available motor braking power during regenerative braking and provides information of the required additional braking by the mechanical friction brakes.

\section{Driving Cycles}

For the purpose of quantitative validation of the developed plant models, the federal test procedure (FTP) city drive cycle is used. This driving cycle is mainly selected for the fuel-economy related validation. The Highway (HWY) fuel economy driving cycle, which represents a mixture of rural and interstate highway driving, is used to simulate typical longer trips in free-flowing traffic with no stops. Also, the high aggressive acceleration driving schedule (US06) is used. Finally the New European Driving Cycle (NEDC), which is performed on a chassis dynamometer for emission testing and fuel consumption, to represent the typical usage of a car in Europe.

First, the conventional ICE vehicle model is run with the aforementioned ICE and vehicle specifications using the selected driving cycles and the most interested outputs were plotted to be used in the comparative analysis. Then the three different 
HEV powertrain models were run using the same ICE parameters' values and the HEV components specs using the same selected driving cycles, and the same outputs were extracted.

\section{COMPARATIVE ANALYSIS}

For the purpose of performance comparisons, several numerical simulations are carried out considering different powertrain configurations namely; conventional, series, parallel, and series-parallel HEVs. While the drivetrain architecture is different for each case, the ICE and EM components have the same technical specifications. For each drivetrain configuration, the numerical simulation is carried out in the GTSuite environment considering the aforementioned four standard driving cycles namely; FTP city driving cycle, HWY driving cycle, high acceleration driving schedule US06, and the NEDC driving cycle.

\section{Simulation Results}

Several simulations are carried out to investigate both the fuel economy and energy analysis of the aforementioned developed powertrains models. First, fuel economy is calculated by employing similar powertrain control strategy for each model. Then, a comprehensive energy analysis is conducted to each powertrain, which explains the reasons for the fuel economy differences among the different architectures.

The simulation results are categorized and presented in three groups as follows:

- The first group is illustrated in Fig. 6 to 9 and demonstrates the fuel consumption rate in $(\mathrm{g} / \mathrm{sec})$ for different drivetrain layouts during HWY driving cycle and considering the battery SOC.

- The second group is illustrated in Fig. 10 to 13 and demonstrates the corresponding vehicle power demand, engine power and the traction motor power produced to follow the HWY driving cycle.

- The third group is illustrated in Fig. 14 to 17 and demonstrates the associated chemical energy depleted from the ICE, and the electrical energy depleted or stored in the battery pack.

For the simulated series HEV powertrain, it is clear that the vehicle's behavior obeys the control strategy, where the ICE/generator set provides more power to run the motor and charge the battery pack whenever the battery SOC hits its lower limit and stops whenever it hits its upper limit, but during the HWY driving cycle and due to the high power demand because of the required high vehicle speed, the ICE is running much longer periods as shown in Fig. 7, and Fig. 11.

For the simulated parallel HEV powertrain, it is clear that the vehicle behavior follows the control strategy during the HWY driving cycle, where the EM acts as propulsion motor during starting and as a generator for charging the batteries during regenerative braking mode, and assists the ICE during high power demand periods. While the ICE is switched off when the vehicle stops and switched on again when the vehicle starts moving after hitting its preset starting speed and provides power to propel the vehicle in hybrid mode as shown in Fig. 8, and Fig. 12. 
For the simulated series-parallel HEV powertrain, the developed model follows the previously explained EMS, where the ICE provides power under certain conditions, either the required speed exceeds the switching speed or the battery SOC drops under its minimum limit, otherwise the EM provides the demanded power to follow the prescribed driving cycle speed as shown in Fig. 9, and Fig. 13.

\section{Energy Analysis}

Energy analysis considers the energy usage and losses breakdown of the entire vehicle, powertrain and driveline system, thus allowing for a global view of the energy management problem and the potential improvements for each HEV design.

The overall energy analysis for different drivetrain layouts and different driving cycles is summarized in Table 3 . The total chemical energy depleted during each driving cycle in [KJ] is a combination of the fuel energy expend and the amount of added penalty. The added penalty arises from the conversion of chemical energy required to regain the battery SOC at the end of the driving cycle.

According to the results of the conventional ICE powertrain model, it is required to spend approximately 65,902 [KJ], and 37,907 [KJ] to run the FTP, and the NEDC driving cycles respectively. From the results comparison, it is clear that using different hybrid drivetrains layouts is significantly saving some energy percentages. This improvement is resulting from the capturing of energy via the regenerative braking during the frequent stops of the city driving profiles.

It should be noted that, according to the simulated results, the series HEV powertrain expends much more energy than the conventional powertrain during the HWY \& US06 driving cycles, $83 \%$ \& $31 \%$ more respectively. This can be referred to the lack of energy regeneration, and using the ICE/Gen set to provide power to the traction motor and recharge the battery pack instead.

\section{Fuel Economy}

For different HEV configurations and considering different driving cycles, the conventional ICE vehicle model shows a lower fuel economy in (miles per gallon). Additionally, the series HEV shows a lower fuel economy during HWY driving cycle which may be due to the excess fuel energy used to power the traction motor and charge the battery for a long period of the driving cycle. On contrary, the seriesparallel HEV model shows the best significant fuel economy for all the driving cycles.

The Brake Specific Fuel Consumption (BSFC) of the ICE, expressed in $[\mathrm{g} / \mathrm{kw} . \mathrm{hr}]$, applies to the distance of the entire driving cycle leading to fuel consumption in $[\mathrm{g} / \mathrm{km}]$. According to the model outputs shown in

Table 3, the series-parallel and the parallel powertrain have better fuel economy for both city and highway driving cycles, while the series powertrain is superior only in frequent stop and go driving. Fig. 18 compares powertrain fuel economies with the different driving cycles. 


\section{CONCLUSIONS}

This paper presented advanced models for different layouts of powertrain which are recently employed in modern hybrid electric vehicles. These models are developed using GT-SUITE software which incorporates a versatile set of tools for the simulation of vehicles with Hybrid-Electric drivelines, as well as the relevant control strategies. In addition, fairly comparisons are carried out to assess the fuel consumption rates, powers and the depleted energies considering four standard driving cycles. The paper results show the positive outcomes from implementing hybrid-electric drivelines in particular the fuel economy improvement. While these findings conform to natural intuition and well known published research, the model provides a quantification of the fuel consumption rates and corresponding vehicle power demand and depleted energy, which is yet to be verified experimentally. 


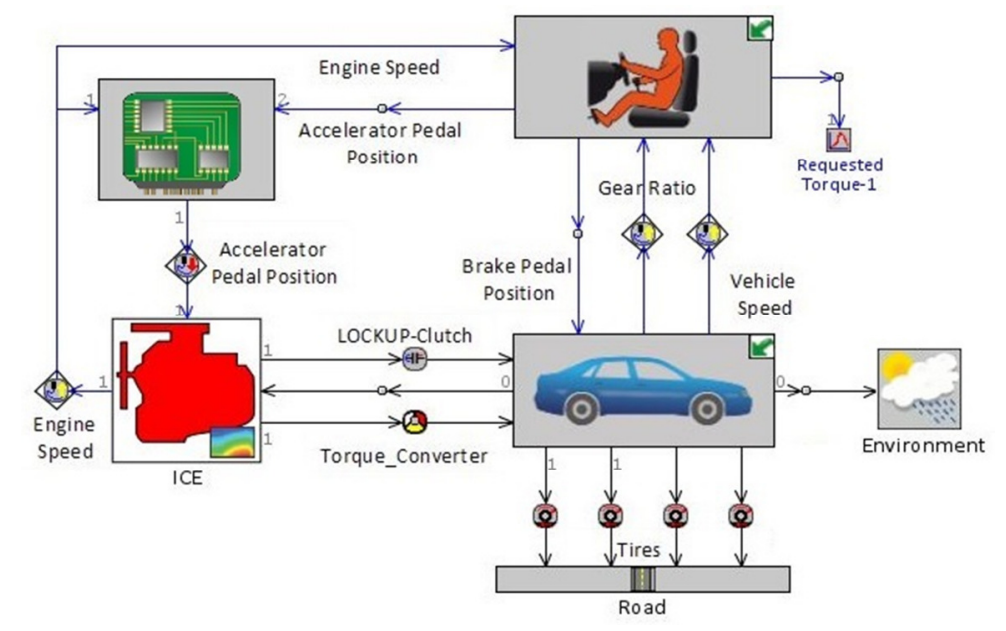

Fig. 1. Conventional power planet model

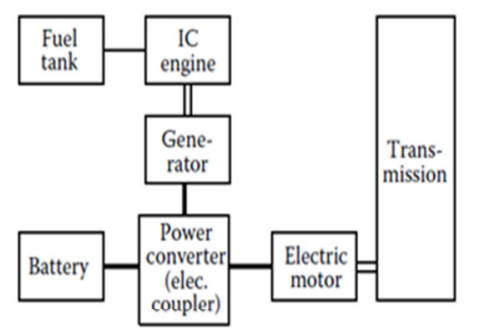

Series HEV

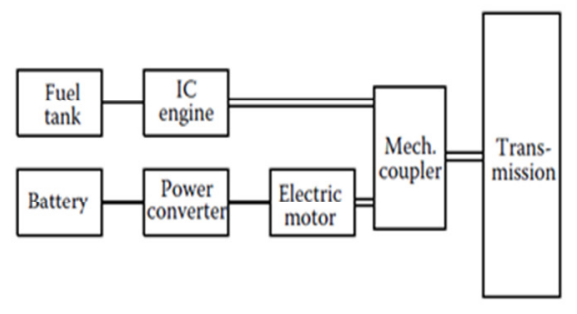

Parallel HEV



Combined HEV

Fig. 2. HEV functional block diagram

Table 1. Conventional ICE and vehicle specifications

\begin{tabular}{|c|c|c|c|c|}
\hline \multicolumn{5}{|c|}{ Engine Specifications } \\
\hline Max Torque & \multicolumn{2}{|c|}{225 Nm @ 3000 rpm } & Min Operating Speed & $500 \mathrm{rpm}$ \\
\hline Max Power & \multicolumn{2}{|c|}{127 HP @ 4500 rpm } & Engine Idle Speed & 800 rpm \\
\hline Total Displacement & \multicolumn{2}{|l|}{$2.0 \mathrm{~L}$} & \multirow{2}{*}{ Max Engine Fueling Speed } & \multirow{2}{*}{$6000 \mathrm{rpm}$} \\
\hline Engine Inertia & $0.6 \mathrm{kgm}^{2}$ & & & \\
\hline \multicolumn{5}{|c|}{ Vehicle Specifications } \\
\hline Vehicle weight & $2000 \mathrm{~kg}$ & & Vehicle Drag coeff. & 0.31 \\
\hline \multirow{2}{*}{$\begin{array}{l}\text { Vehicle rolling } \\
\text { resistance coeff. }\end{array}$} & \multirow{2}{*}{\multicolumn{2}{|c|}{0.01}} & Vehicle Frontal Area & $2.5 \mathrm{~m}^{2}$ \\
\hline & & & Wheel base & $2 \mathrm{~m}$ \\
\hline \multicolumn{5}{|c|}{ Transmission Gear Ratios } \\
\hline Gear \#1 & Gear \#2 & Gear \# & Gear \#4 & Gear \#5 \\
\hline 3.5 & 2.1 & 1.4 & 1 & 0.72 \\
\hline
\end{tabular}

Table 2. HEV model specifications

\begin{tabular}{|l|c|l|c|}
\hline \multicolumn{4}{|c|}{ Battery / Inverter Specifications } \\
\hline Batt. max. charge current & $50 \mathrm{~A}$ & Batt. capacity & $23 \mathrm{Ah}$ \\
\hline Batt. max. discharge current & $100 \mathrm{~A}$ & SOC max. limit & 0.7 \\
\hline Batt. max. voltage & $400 \mathrm{~V}$ & SOC min. limit & 0.5 \\
\hline Batt. min. voltage & $200 \mathrm{~V}$ & & \\
\hline
\end{tabular}






Fig. 3. Series HEV planet model

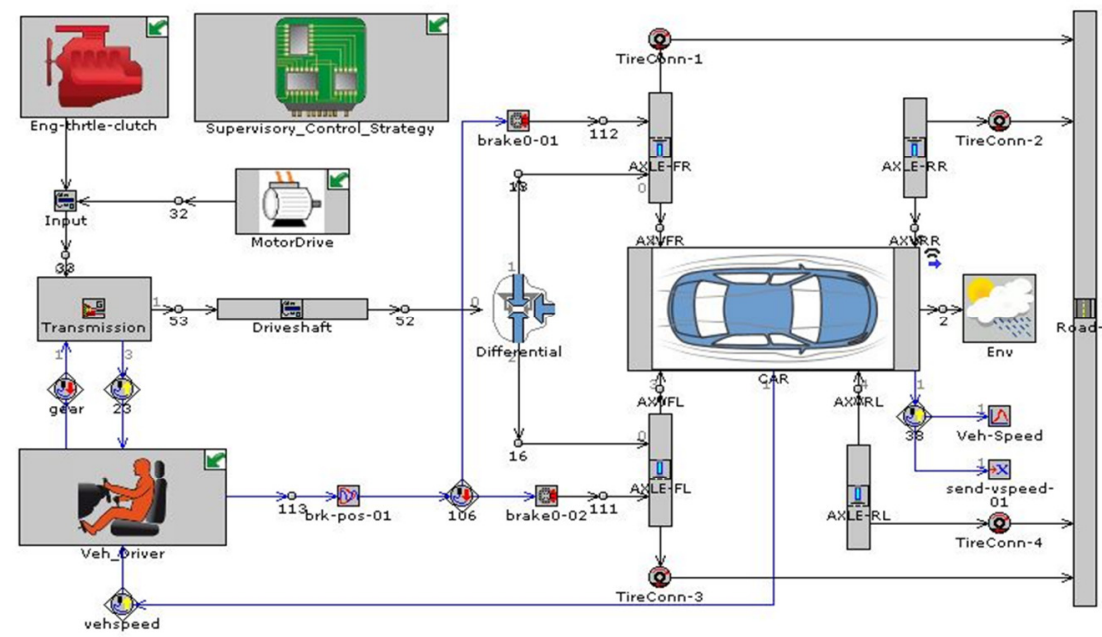

Fig. 4. Parallel HEV planet model



Fig. 5. Series-Parallel HEV planet model 


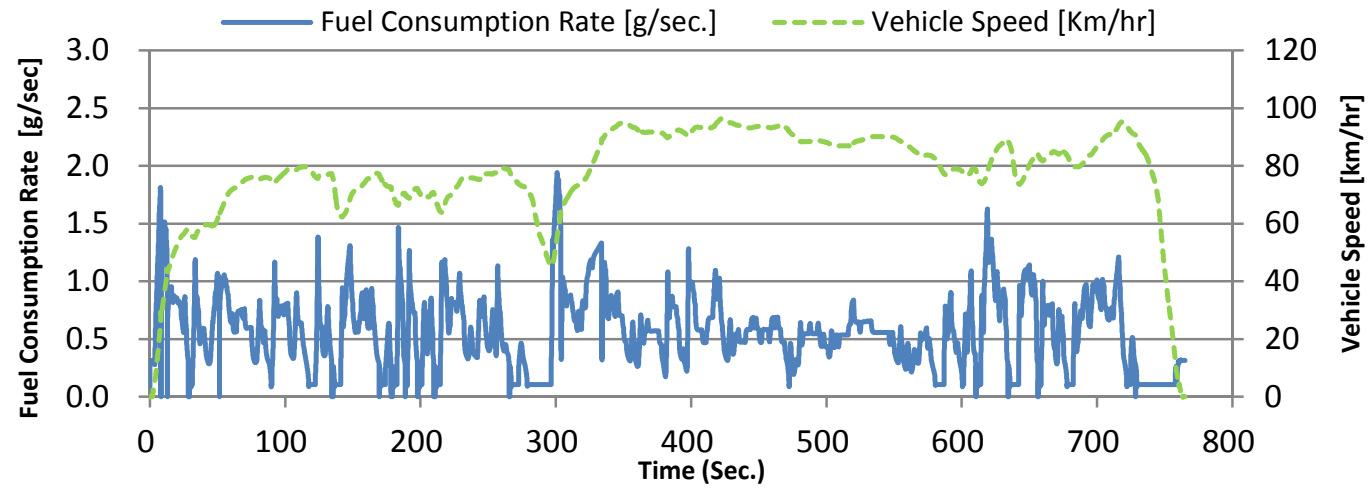

Fig. 6. ICE Vehicle undergoing HWY driving cycle

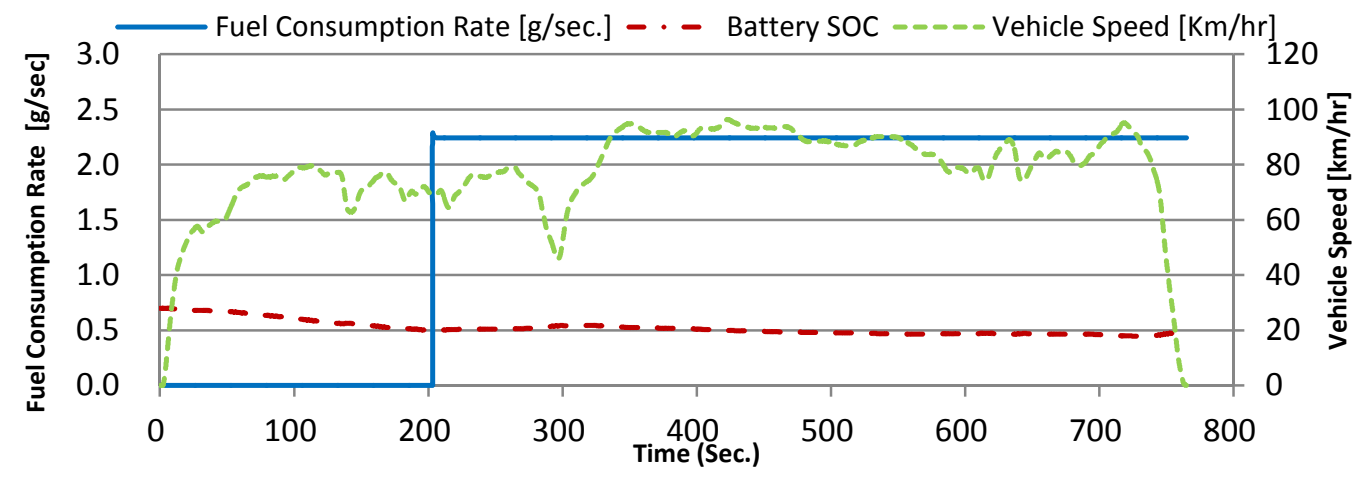

Fig. 7. Series HEV undergoing HWY driving cycle



Fig. 8. Parallel HEV undergoing HWY driving cycle

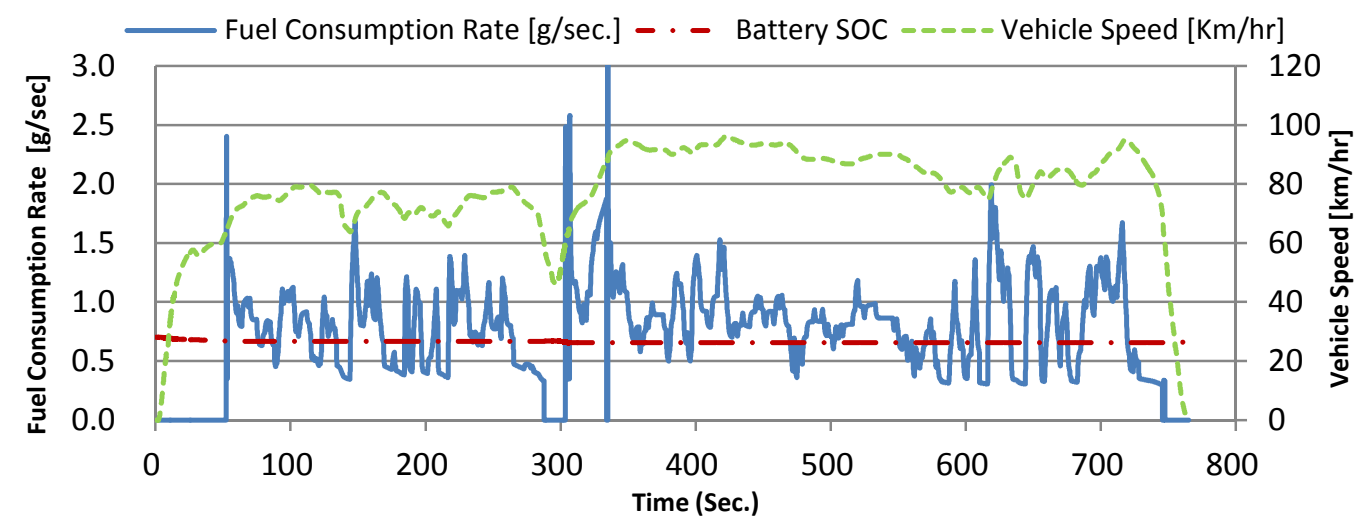

Fig. 9. Series-Parallel HEV undergoing HWY driving cycle 


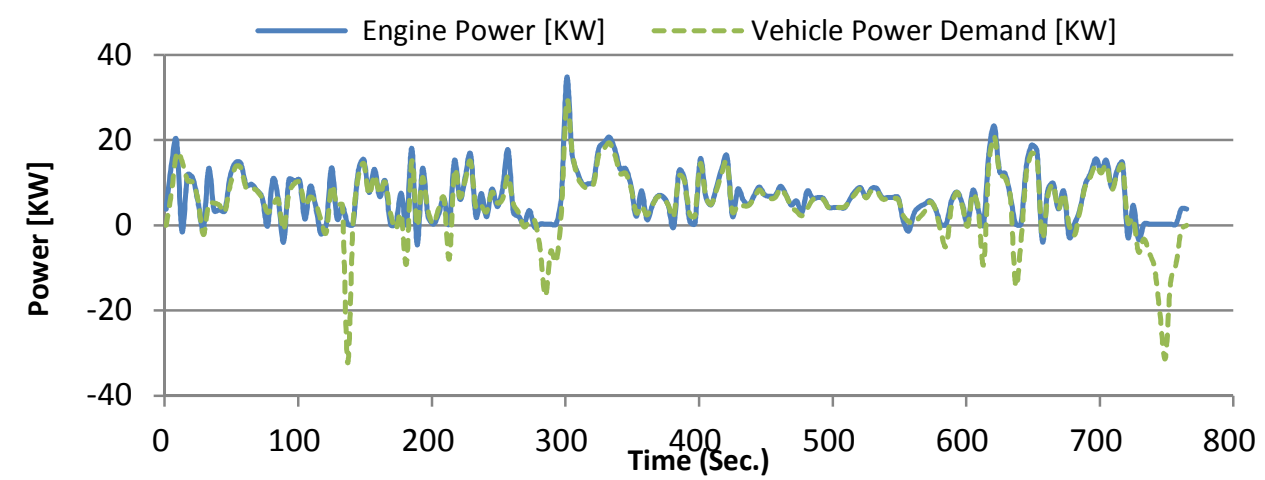

Fig. 10. Power demand \& power production during HWY driving cycle in ICE vehicle

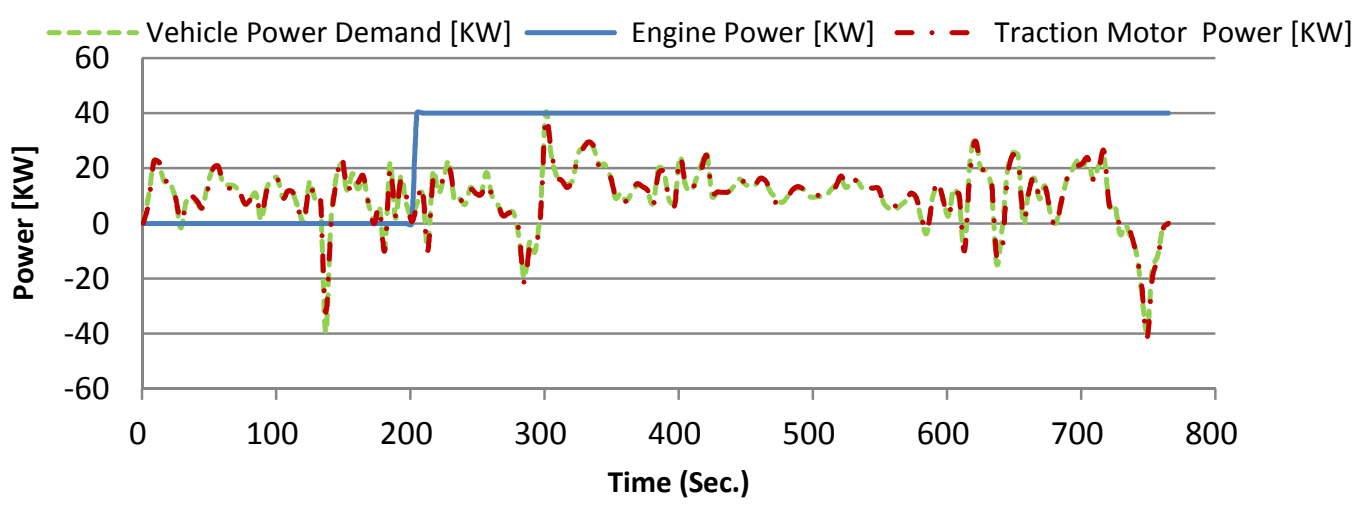

Fig. 11. Power demand \& power production during HWY driving cycle in series HEV

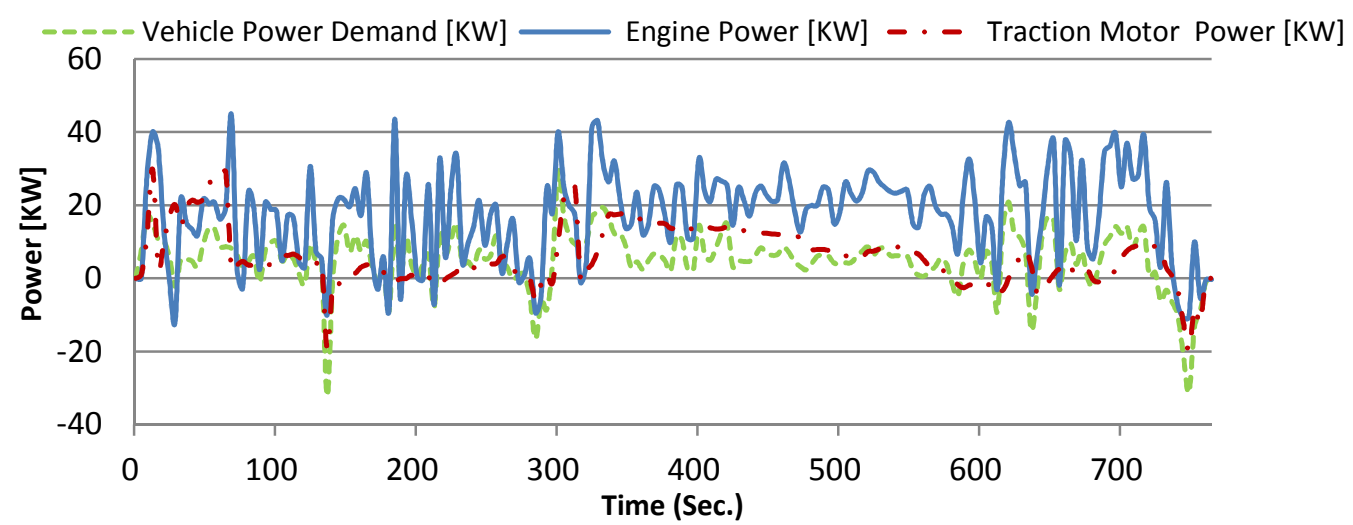

Fig. 12. Power demand \& power production during HWY driving cycle in parallel HEV

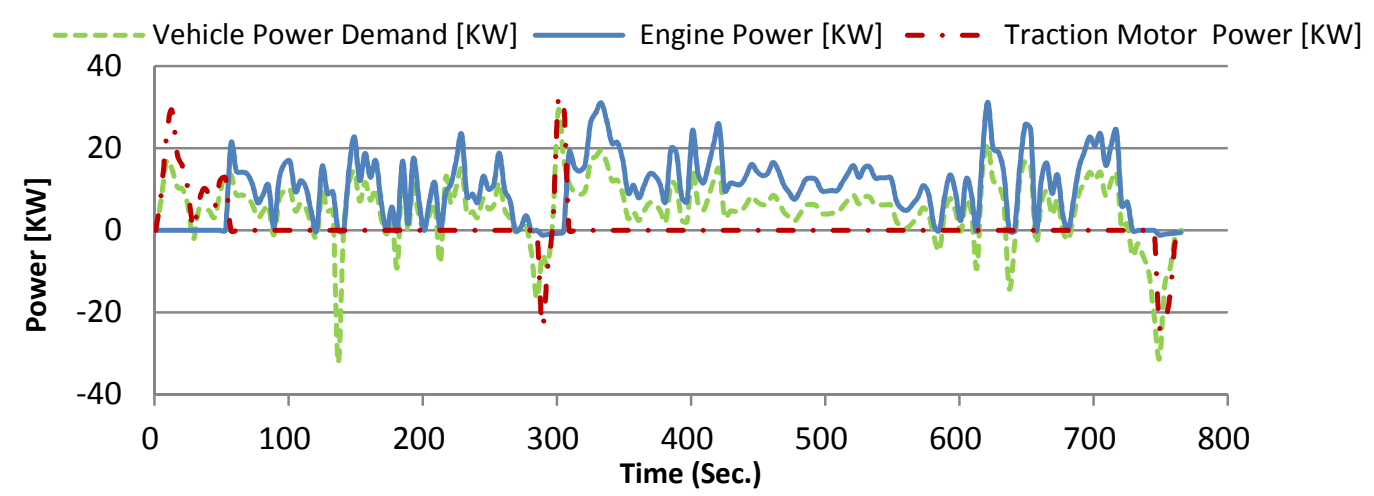

Fig. 13. Power demand \& power production during HWY driving cycle in seriesparallel HEV 


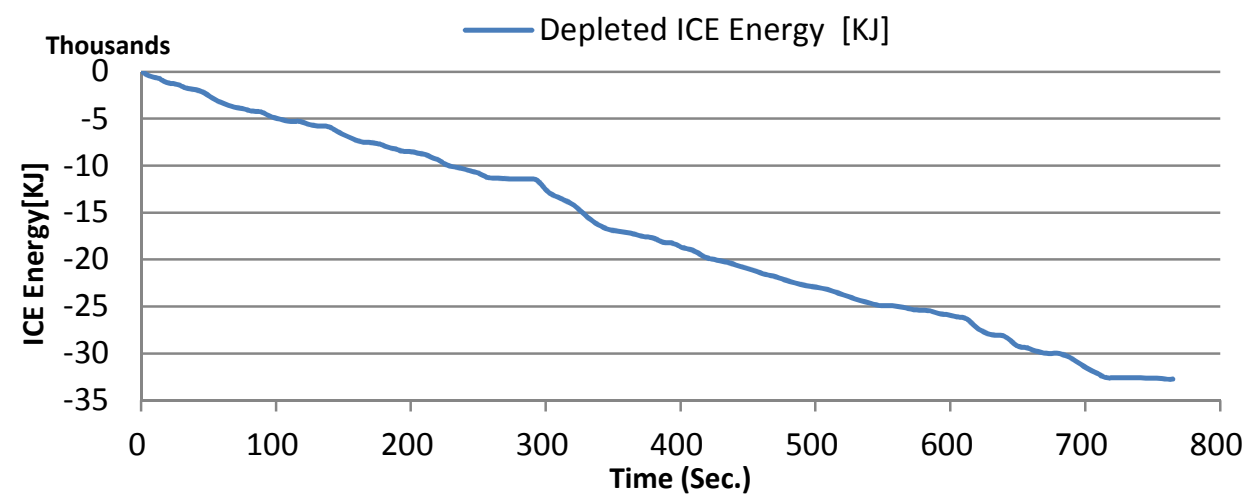

Fig. 14. Depleted energy during HWY driving cycle in ICE vehicle



Fig. 15. Depleted and stored energy during HWY driving cycle in series HEV

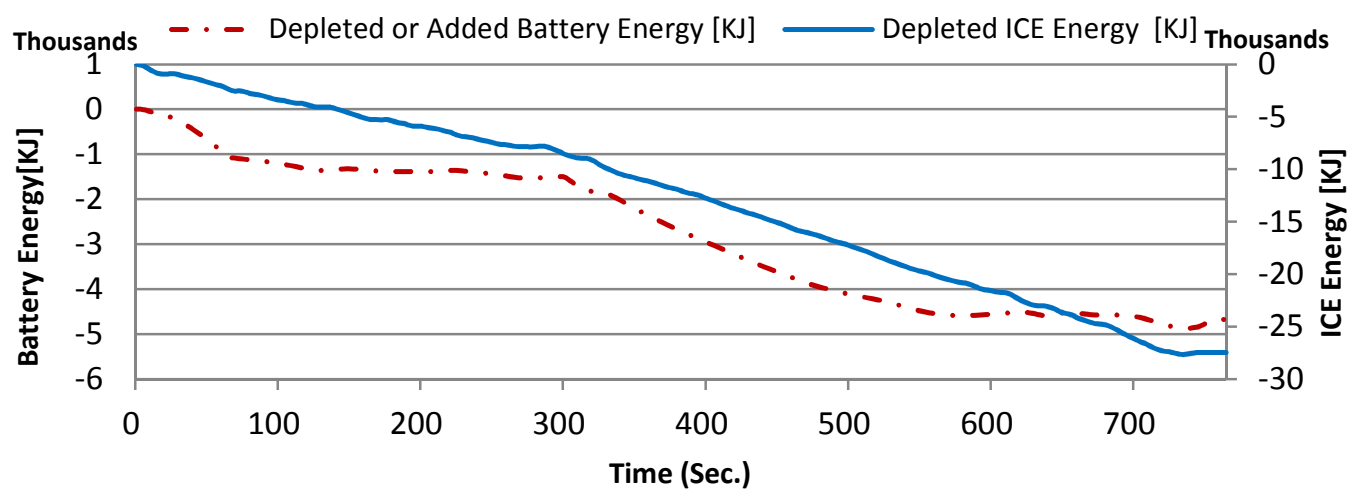

Fig. 16. Depleted and stored energy during HWY driving cycle in parallel HEV



Fig. 17. Depleted and stored energy during HWY driving cycle in series-parallel HEV 
Table 3. Different models outputs

\begin{tabular}{|c|c|c|c|c|c|c|c|c|c|}
\hline $\begin{array}{l}\vec{山} \\
\text { Oे } \\
\sum\end{array}$ & 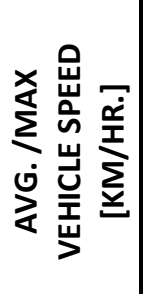 & 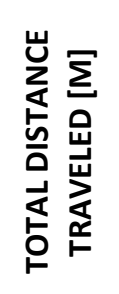 & 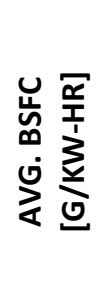 & 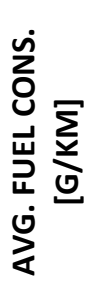 & 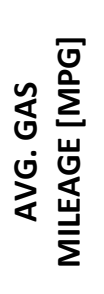 & 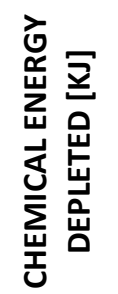 & 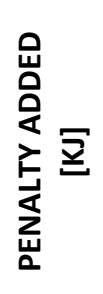 & 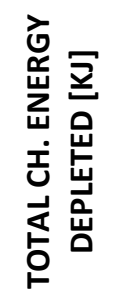 & 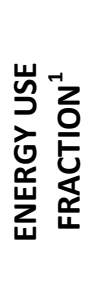 \\
\hline \multicolumn{10}{|c|}{ FTP CITY DRIVING CYCLE } \\
\hline CONVENTIONAL & \multirow{4}{*}{$34 / 91$} & 17,648 & 396.2 & 84.6 & 21 & 65,902 & 0 & 65,902 & 1 \\
\hline SERIES HEV & & 17,746 & 202 & 66.1 & 26.9 & 51,790 & $-3,407$ & 48,383 & 0.73 \\
\hline PARALLEL HEV & & 17,640 & 169.5 & 59.5 & 29.9 & 45,588 & 3,868 & 49,456 & 0.75 \\
\hline COMBINED HEV & & 17,637 & 219.2 & 36.4 & 48.9 & 28,335 & 444 & 28,779 & 0.44 \\
\hline \multicolumn{10}{|c|}{ HWY DRIVING CYCLE } \\
\hline CONVENTIONAL & \multirow{4}{*}{$77 / 96$} & 16,490 & 395.1 & 59.3 & 29.5 & 32,737 & 0 & 32,737 & 1 \\
\hline SERIES HEV & & 16,502 & 202 & 76.4 & 23.3 & 55,694 & 4,235 & 59,929 & 1.83 \\
\hline PARALLEL HEV & & 16,480 & 160.6 & 37.7 & 47.1 & 27,456 & 4,672 & 32,128 & 0.98 \\
\hline COMBINED HEV & & 16,514 & 261.1 & 33.9 & 52.5 & 24,686 & 866 & 25,552 & 0.78 \\
\hline \multicolumn{10}{|c|}{ US06 DRIVING CYCLE } \\
\hline CONVENTIONAL & \multirow{4}{*}{$76 / 130$} & 12,764 & 336 & 73.5 & 24.2 & 41,424 & 0 & 41,424 & 1 \\
\hline SERIES HEV & & 12,580 & 202 & 80.5 & 22.1 & 44,737 & 9,670 & 54,407 & 1.31 \\
\hline PARALLEL HEV & & 12,651 & 150 & 50 & 35.6 & 27,920 & 3,222 & 31,142 & 0.75 \\
\hline COMBINED HEV & & 12,488 & 242.2 & 40.4 & 44 & 22,270 & 3,301 & 25,571 & 0.62 \\
\hline \multicolumn{10}{|c|}{ NEDC DRIVING CYCLE } \\
\hline CONVENTIONAL & \multirow{4}{*}{$33 / 120$} & 10,972 & 420 & 78.2 & 22.7 & 37,907 & 0 & 37,907 & 1 \\
\hline SERIES HEV & & 11,008 & 202 & 33.7 & 52.8 & 16,378 & 4,738 & 21,117 & 0.56 \\
\hline PARALLEL HEV & & 10,965 & 174 & 51.9 & 34.2 & 25,150 & 4,568 & 29,718 & 0.78 \\
\hline COMBINED HEV & & 11,004 & 246.2 & 23.9 & 58.2 & 10,142 & 3,107 & 13,249 & 0.35 \\
\hline
\end{tabular}

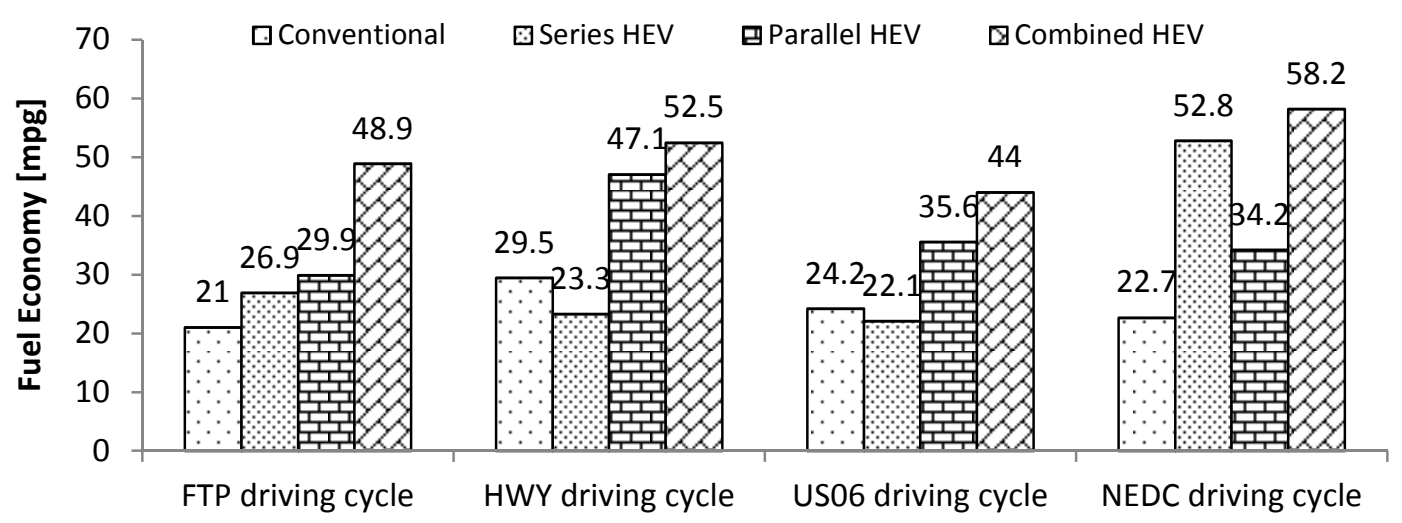

Fig. 18. Fuel economy of different powertrain models

\footnotetext{
${ }^{1}$ Energy use compared to ICE only powertrain for a specific driving cycle
} 


\section{REFRENCES}

[1] M. Ehsani, Y. Gao, and A. Emadi, "Modern Electric, Hybrid Electric, and Fuel Cell Vehicles: Fundamentals, Theory, and Design", CRC Press, (2010).

[2] I. Husain, "Electric and Hybrid Vehicles: Design Fundamentals", CRC Press, (2003).

[3] L. Guzzella and A. Sciarretta, "Vehicle Propulsion Systems: Introduction to Modeling and Optimization", Springer, (2005).

[4] X. Zhang and C. Mi, "Vehicle Power Management: Modeling, Control and Optimization", 1st Edition, Springer, (2011).

[5] C.C. Lin, Z. Filipi, Y. Wang, L. Louca, H. Peng, D. Assanis, and J. Stein, "Integrated, Feed-Forward Hybrid Electric Vehicle Simulation in SIMULINK and its Use for Power Management Studies", SAE International, Warrendale, PA, 2001-01-1334, (2001).

[6] J. Liu, H. Peng and Z. Filipi, "Modeling and Control Analysis of Toyota Hybrid System", IEEE/ASME International Conference on Advanced Intelligent Mechatronics, pp. 134-139, (2005).

[7] T. Markel, A. Brooker, T. Hendricks, V. Johnson, K. Kelly, B. Kramer, M. O'Keefe, S. Sprik, and K. Wipke, "ADVISOR: a systems analysis tool for advanced vehicle modeling", Journal of Power Sources, Vol. 110, No. 2, pp. 255-266, Aug. (2002).

[8] A. Rousseau and M. Pasquier, "Validation of a hybrid modeling software (PSAT) using its extension for prototyping (PSAT-PRO)", Proceedings of the 2001 Global Powertrain Congress, Detroit, MI, USA, pp. 1-9, (2001).

[9] D. W. Gao, C. Mi, and A. Emadi, "Modeling and Simulation of Electric and Hybrid Vehicles", Proceedings of the IEEE, Vol. 95, No. 4, pp. 729-745, Apr. (2007).

[10] F. Millo, L. Rolando, and M. Andreat, "Numerical Simulation for Vehicle Powertrain Development", In Numerical Analysis - Theory and Application, J.Awrejcewicz, Ed. InTech, (2011).

[11] K. Chau and Y. Wong, "Overview of power management in hybrid electric vehicles", Energy Conversion and Management, Vol. 43, No. 15, pp. 19531968, Oct. (2002).

[12] A. I. Antoniou, J. Komyathy, J. Bench, and A. Emadi, "Modeling and Simulation of Various Hybrid-Electric Configurations of the High-Mobility Multipurpose Wheeled Vehicle (HMMWV)", IEEE Transactions on Vehicular Technology, Vol. 56, No. 2, pp. 459-465, Mar. (2007). 